\title{
OP 030 PLANNING FOR THE FUTURE WITH PATIENTS WHO HAVE ADVANCED HEART DISEASE: DESIGNING A RANDOMISED CLINICAL TRIAL OF ANTICIPATORY CARE
}

Gill Highet, ${ }^{1}$ Sarah Cudmore, ${ }^{2}$ Shirley Robertson, ${ }^{2}$ Scott A Murray, ${ }^{3}$ Kirsty Boyd, ${ }^{1}$ Martin A Denvir ${ }^{2}{ }^{1}$ Department of Palliative Care, Royal Infirmary of Edinburgh, Scotland; ' ${ }^{2}$ Edinburgh Heart Centre, Royal Infirmary of Edinburgh, Scotland; ${ }^{3}$ Primary Palliative Care Research Group, University of Edinburgh, Scotland

10.1136/bmjspcare-2014-000654.30

Background Many patients with advanced heart disease have a limited prognosis despite optimal medical, surgical and device therapy. These patients rarely receive coordinated, holistic care that acknowledges their individual needs and those of their informal carers.

Aims This study assesses the feasibility of a randomised controlled trial of a complex intervention that incorporates ongoing, holistic care with anticipatory care planning for people with advanced heart disease.

Methods We conducted 11 interviews with a diverse sample of healthcare professionals and 2 focus groups comprising 12 patients and 5 carers. Participants were given a previously developed "Future Care Plan" and details of the trial before the interviews/ focus groups. These acted as focal points for discussion. Data were transcribed and analysed using standard software to extract themes related to the rationale and design of a clinical trial of anticipatory care planning in advanced heart disease. 
Results Patients and carers highlighted fragmentation of services, difficulty in accessing specialist care and inadequate time for future planning and preparation as key barriers to holistic care. A patient-held plan was welcomed. Healthcare professionals identified challenges related to uncertainty of prognosis, explaining mortality-risk to patients, and switching from curative to palliative approaches. These data informed patient selection (a combination of prognostic tools and needs for additional supportive care), the intervention (cardiologist review and nurse-led support delivered though a patient-centred care plan), and clinical trial outcomes that are robust but measurable in a frail population.

Conclusions Patients, carers and healthcare professionals share a number of common concerns in relation to providing high quality care for people with advanced heart disease. The findings of this exploratory study are informing the implementation of a phase II randomised clinical trial of an holistic, anticipatory care planning intervention for patients with advanced heart disease in line with good practice in end of life care. 